\title{
The Fundamental Solution of a Modified Oseen Problem
}

\author{
R. Farwig, A. Novotný and M. Pokorný
}

\begin{abstract}
We study asymptotic properties of the fundamental solution to an Oseen-type system coming from fluid mechanics. We show that the solution has similar anisotropic structure near infinity as the fundamental solution to the (classical) Oseen problem. We also study integral operators with kernels representing the second gradient of the fundamental solution.

Keywords: Systems of partial differential equations, fundamental solutions, Oseen problem, Fourier transform, Fourier multipliers
\end{abstract}

AMS subject classification: 35 A 08, 35 Q 35, 42 B 15

\section{Introduction}

This paper is devoted to the study of the Oseen-type problem

$$
\left.\begin{array}{r}
-\Delta u+\mu \frac{\partial^{2} u}{\partial x_{1}^{2}}+\beta \frac{\partial u}{\partial x_{1}}+\nabla \pi=f \\
\nabla \cdot u=0
\end{array}\right\},
$$

$0 \leq \mu<1$, called here the modified Oseen problem. This problem appears e.g. in the study of asymptotic properties of solutions to the system of partial differential equations describing the steady flow of certain classes of incompressible viscoelastic fluids past an obstacle. In this case, the velocity $v$ and the pressure $p$ satisfy

$$
\left.\begin{array}{rl}
-\eta \Delta v+\varrho(v \cdot \nabla) v+\nabla \pi= & \varrho f+\nabla \cdot[F(\nabla v, T)-\lambda \varrho((v \cdot \nabla) v) \otimes v \\
& \left.+\lambda \varrho f \otimes v+\lambda p(\nabla v)^{T}\right] \\
\nabla \cdot v= & 0 \\
p+\lambda(v \cdot \nabla) p= & \pi \\
T+\lambda(v \cdot \nabla) T+G(\nabla v, T)= & 2 \eta D(v)
\end{array}\right\}
$$

R. Farwig: FB Math., Techn. Univ. Darmstadt, Schloßgartenstr. 7, D-64287 Darmstadt A. Novotný: Univ. de Toulon et du Var, Lab. A.N.L.A., B.P. 132, 83957 La Garde, France M. Pokorný: Palacký Univ., Dept. Math. Anal. \& Appl. Math., Tomkova 40, 77200 Olomouc, Czech Rep.; supported by the Grant of Palacký Univ. No. 31103006.

farwig@mathematik.tu-darmstadt.de; novotny@univ-tln.fr; pokorny@risc.upol.cz 
where

$F(\cdot, \cdot), G(\cdot, \cdot)$ are bilinear functions

$T$ is the extra stress tensor

$D$ is the symmetric part of the velocity gradient

$f$ is a given function (external force)

$\varrho$ is the constant density

$\lambda, \eta$ are positive constants.

We assume system (0.2) to hold in an exterior domain $\Omega \subset \mathbb{R}^{N}(N=2,3)$ and consider the boundary conditions

$$
\left.\begin{array}{rlrl}
v & =0 & & \text { on } \partial \Omega \\
v \rightarrow v_{\infty} & =\beta e_{1} & & \text { as }|x| \rightarrow \infty
\end{array}\right\} .
$$

We may construct a solution to system (0.2) - (0.3) by means of the following procedure. Define the linear operator

$$
\mathcal{M}:(w, s) \rightarrow(u, \pi)
$$

where

$$
\begin{aligned}
-\eta \Delta u+\lambda \varrho \beta^{2} \frac{\partial^{2} u}{\partial x_{1}^{2}}+\varrho \beta \frac{\partial u}{\partial x_{1}}+\nabla \pi & =\varrho f+\nabla \cdot[F(\nabla w, T) \\
& -\lambda \varrho((w \cdot \nabla) w) \otimes w-\varrho w \otimes w \\
& -\lambda \varrho \beta\left(\frac{\partial w}{\partial x_{1}} \otimes w+((w \cdot \nabla) w) \otimes e_{1}\right) \\
& \left.+\lambda \varrho f \otimes\left(w+\beta e_{1}\right)+\lambda p(\nabla w)^{T}\right] \\
\nabla \cdot u= & 0 \\
p+\lambda\left(\left(w+v_{\infty}\right) \cdot \nabla\right) p= & s \\
T+\lambda\left(\left(w+v_{\infty}\right) \cdot \nabla\right) T+G(\nabla w, T)= & 2 \eta D(w)
\end{aligned}
$$

and $u$ satisfies the boundary conditions

$$
\left.\begin{array}{ll}
u \rightarrow 0 & \text { as }|x| \rightarrow \infty \\
u=-\beta e_{1} & \text { on } \partial \Omega
\end{array}\right\} .
$$

Let $(\bar{u}, \bar{\pi})$ be a fixed point of $\mathcal{M}$. Then $(v, p)$, with $v=\bar{u}+v_{\infty}$ and $p$ solution to $(0.4)_{3}$ with $s=\bar{\pi}$ and $w=\bar{u}$, solves the original problem $(0.2)-(0.3)$. We observe that the elliptic part of problem (0.4) is exactly system $(0.1)$, i.e. the modified Oseen problem.

Let us note two peculiarities.

Instead of only constructing solutions to problem $(0.2)-(0.3)$, we also want to study their asymptotic properties near infinity. Otherwise, we could put the term $\lambda \varrho \beta^{2} \frac{\partial^{2}}{\partial x_{1}^{2}}$ 
to the right-hand side of $(0.4)_{1}$ and consider the (classical) Oseen problem instead of the modified one. But in this case we could not get weighted estimates of the solution due to certain loss in the estimates of convolutions with kernels representing the second gradient of the fundamental Oseen tensor (cf. $[1,6]$ or [3]). Therefore, the aim of this paper is to consider the asymptotic structure of the fundamental solution to the modified Oseen problem (0.1). The application of this analysis can be found in [6] or $[4,5]$.

Secondly, at a first glance it seems to be possible to change variables and modify the vector $v$ in such a way that we would get the (classical) Oseen problem. This is not true; the condition $\nabla \cdot v=0$ would be replaced by another condition and we could not treat the problem using the results on the (classical) Oseen problem.

Many properties of solutions to the (classical) Oseen problem are connected with the properties of the fundamental solution to the Oseen problem. The same holds also in the case of the modified version (cf. [6]). We are looking for $\left(\mathcal{O}^{\mu}, e\right)$ such that ${ }^{1)}$

$$
\left.\begin{array}{rl}
{\left[-\Delta+\mu \frac{\partial^{2}}{\partial x_{1}^{2}}+\lambda \frac{\partial}{\partial x_{1}}\right] \mathcal{O}_{i j}^{\mu}(x ; \lambda)+\frac{\partial}{\partial x_{i}} e_{j}(x)} & =\delta_{i j} \delta \\
\frac{\partial \mathcal{O}_{i j}^{\mu}(x ; \lambda)}{\partial x_{i}} & =0
\end{array}\right\}
$$

where $\delta_{i j}$ denotes the Kronecker delta, $\delta$ is the Dirac $\delta$-distribution, $0 \leq \mu<1$, and system (0.6) holds in the sense of distributions.

Throughout the paper we use the standard notation $L^{p}\left(\mathbb{R}^{N}\right)$ for Lebesgue spaces, $\mathcal{D}\left(\mathbb{R}^{N}\right)$ for the set of smooth functions with compact support and $\mathcal{S}$ for the Schwartz class. By $\mathcal{F}(f)=\hat{f}$ we denote the Fourier transform of the function $f$, either in the sense of $L^{2}\left(\mathbb{R}^{N}\right)$ or in the sense of $\mathcal{S}^{\prime}$.

\section{Fundamental solution to the Oseen problem}

Before starting to study problem (0.6), let us recall several properties of the fundamental solution to the (classical) Oseen problem, i.e. problem (0.6) with $\mu=0$. We shall write only $\mathcal{O}$ instead of $\mathcal{O}^{0}$ in this case. As is well known (see, e.g., [2]),

$$
e=\nabla \mathcal{E}(x)
$$

with $\mathcal{E}(x)$ the fundamental solution to the Laplace equation. The tensor $\mathcal{O}$ satisfies the homogeneity property

$$
\mathcal{O}(x, \lambda)=\lambda^{N-2} \mathcal{O}(\lambda x ; 1)
$$

and therefore it is sufficient to study only the case $\lambda=1$.

For small $|x|$,

$$
\mathcal{O}(x ; 1)=\mathcal{S}(x)+\mathcal{R}(x)
$$

1) We use the standard summation convention. 
where $\mathcal{S}$ is the fundamental Stokes tensor (see, e.g., [2]) and in three dimensions ${ }^{2)}$

$$
D^{\alpha} \mathcal{R}(x) \sim|x|^{-|\alpha|} \quad \text { for }|x| \rightarrow 0
$$

for $|\alpha| \geq 0$, while in two dimensions

$$
\begin{aligned}
\mathcal{R}(x) & \sim 1 \\
D^{\alpha} \mathcal{R}(x) & \sim\left\{\begin{array}{ll}
\ln |x| & \text { if }|\alpha|=1 \\
|x|^{-|\alpha|+1} & \text { if }|\alpha| \geq 2
\end{array}\right\}
\end{aligned} \quad \text { for }|x| \rightarrow 0
$$

Recall that

$$
\mathcal{S}_{i j}(x)= \begin{cases}\frac{1}{4 \pi}\left(\delta_{i j} \log \frac{1}{|x|}+\frac{x_{i} x_{j}}{|x|^{2}}\right) & \text { if } N=2 \\ \frac{1}{8 \pi}\left(\frac{\delta_{i j}}{|x|}+\frac{x_{i} x_{j}}{|x|^{3}}\right) & \text { if } N=3 .\end{cases}
$$

Therefore the second derivatives of $\mathcal{S}$ represent Calderón-Zygmund singular integral kernels, i.e. there exists $C=C(p, N)$ such that

$$
T: \mathcal{D}\left(\mathbb{R}^{N}\right) \mapsto \mathcal{D}^{\prime}\left(\mathbb{R}^{N}\right), \quad(T f)(x)=\int_{\mathbb{R}^{N}} \frac{\partial \mathcal{S}_{k l}(x-y)}{\partial x_{i}} \frac{\partial f(y)}{\partial y_{j}} d y
$$

satisfies

$$
\|T f\|_{L^{p}} \leq C\|f\|_{L^{p}}
$$

for all $f \in \mathcal{D}\left(\mathbb{R}^{N}\right)$ and $1<p<\infty$. Thus the operator $T$ can be continuously extended onto $L^{p}\left(\mathbb{R}^{N}\right)$. Moreover, it can be shown that

$$
(T f)(x)=\text { v.p. } \int_{\mathbb{R}^{N}} \frac{\partial^{2} \mathcal{S}_{k l}\left(x^{\prime}-y\right)}{\partial x_{i} \partial x_{j}} f(y) d y+c_{i j k l} f(x) .
$$

Define $s(x)=|x|-x_{1}$. For $|x| \rightarrow \infty$ we have in three space dimensions

$$
\begin{aligned}
\mathcal{O}(x ; 1) & \sim|x|^{-1}(1+s(x))^{-1} \\
D^{\alpha} \mathcal{O}(x ; 1) & \sim|x|^{-1-\frac{|\alpha|}{2}}(1+s(x))^{-1-\frac{|\alpha|}{2}} \quad(|\alpha| \geq 1) .
\end{aligned}
$$

The derivatives with respect to the first variable decay faster; more precisely,

$$
D^{\alpha} \mathcal{O}(x ; 1) \sim|x|^{-1-\alpha_{1}-\frac{|\alpha|-\alpha_{1}}{2}}(1+s(x))^{-1-\frac{|\alpha|-\alpha_{1}}{2}} \quad(|\alpha| \geq 1)
$$

2) Throughout this paper, $f \sim g$ for $|x| \rightarrow 0$ means that there exists $\mathcal{U}_{\varepsilon}(0)$, a neighborhood of 0 , and $C_{1}, C_{2} \in \mathbb{R}$ such that $C_{1}|f(x)| \leq g(x) \leq C_{2}|f(x)|$ for $x \in \mathcal{U}_{\varepsilon}(0)$; analogously for $|x| \rightarrow \infty$. 
In two space dimensions the structure is even more complicated. Here we have for $|x| \rightarrow \infty\left(\right.$ recall that $\left.\mathcal{O}_{i j}(x ; 1)=\mathcal{O}_{j i}(x ; 1)\right)$

$$
\begin{aligned}
\mathcal{O}_{11}(x ; 1) & \sim|x|^{-\frac{1}{2}}(1+s(x))^{-\frac{1}{2}} \\
\mathcal{O}_{i j}(x ; 1) & \sim|x|^{-1}((i, j) \neq(1,1)) \\
\frac{\partial \mathcal{O}_{11}(x ; 1)}{\partial x_{2}} & \sim|x|^{-1}(1+s(x))^{-1} \\
\frac{\partial \mathcal{O}_{11}(x ; 1)}{\partial x_{1}}, \frac{\partial \mathcal{O}_{12}(x ; 1)}{\partial x_{2}} & \sim|x|^{-\frac{3}{2}}(1+s(x))^{-\frac{1}{2}} \\
\frac{\partial \mathcal{O}_{12}(x ; 1)}{\partial x_{1}}, \frac{\partial \mathcal{O}_{22}(x ; 1)}{\partial x_{i}} & \sim|x|^{-2}(i=1,2) \\
\frac{\partial^{2} \mathcal{O}_{11}(x ; 1)}{\partial x_{2}^{2}} & \sim|x|^{-\frac{3}{2}}(1+s(x))^{-\frac{3}{2}} \\
\frac{\partial^{2} \mathcal{O}_{i j}(x ; 1)}{\partial x_{k} \partial x_{l}} & \sim|x|^{-2}(1+s(x))^{-1} \quad((i, j, k, l) \neq(1,1,2,2)) .
\end{aligned}
$$

By analogy with the three-dimensional case the derivatives with respect to the first variable decay faster. Especially,

$$
\frac{\partial^{2} \mathcal{O}_{i j}(x)}{\partial x_{1}^{2}} \sim|x|^{-\frac{5}{2}}(1+s(x))^{-\frac{1}{2}} \quad(i, j=1,2) .
$$

Our aim is to show similar properties also for the modified problem (0.6).

\section{Fundamental solution to the modified Oseen problem}

We shall search $\mathcal{O}^{\mu}$ in the form

$$
\mathcal{O}^{\mu}(x ; \lambda)=\mathcal{O}(x ; \lambda)+E^{\mu}(x ; \lambda)
$$

where $\mathcal{O}$ is the (classical) fundamental Oseen tensor and

$$
\left[\Delta-\mu \frac{\partial^{2}}{\partial x_{1}^{2}}-\lambda \frac{\partial}{\partial x_{1}}\right] E_{i j}^{\mu}=\mu \frac{\partial^{2} \mathcal{O}_{i j}}{\partial x_{1}^{2}}
$$

in the sense of distributions. As in the Stokes and also classical Oseen problem the fundamental pressure $e$ is given by $e_{j}(x)=\frac{\partial \mathcal{E}(x)}{\partial x_{j}}$. Therefore it is sufficient to study $E^{\mu}$, the solution to (2.2). We further need

$$
\frac{\partial E_{i j}^{\mu}}{\partial x_{i}}=0
$$

in the sense of distributions, which will be proved in Lemma 2.2 below. 
First we consider the fundamental solution to (2.2), i.e.

$$
\left[\Delta-\mu \frac{\partial^{2}}{\partial x_{1}^{2}}-\lambda \frac{\partial}{\partial x_{1}}\right] E^{*}=\delta
$$

in the sense of distributions. For these reasons define

$$
X_{i}=\frac{x_{i}}{\sqrt{1-\mu \delta_{i 1}}} \quad \text { and } \quad \bar{\lambda}=\frac{\lambda}{\sqrt{1-\mu}} .
$$

Lemma 2.1. The fundamental solution to (2.4) is given by

$$
E^{*}(x ; \lambda)= \begin{cases}\frac{-1}{2 \pi \sqrt{1-\mu}} K_{0}\left(\frac{\bar{\lambda} R}{2}\right) e^{\bar{\lambda} X_{1} / 2} & \text { if } N=2 \\ \frac{-1}{4 \pi \sqrt{1-\mu}} \frac{e^{-\bar{\lambda} s(X) / 2}}{R} & \text { if } N=3 .\end{cases}
$$

Here $X$ and $\bar{\lambda}$ are defined by (2.5), $R=|X|, s(X)=R-X_{1}$, and $K_{0}$ denotes a modified Bessel function of the second kind satisfying

$$
K_{0}(z)= \begin{cases}-\ln z+O(1) & \text { for }|z| \rightarrow 0 \\ \left(\frac{\pi}{2 z}\right)^{1 / 2} e^{-z}\left(1+O\left(\frac{1}{|z|}\right)\right) & \text { for }|z| \rightarrow \infty .\end{cases}
$$

Proof. Using (2.5) we transform (2.4) into

$$
\left(\Delta-\bar{\lambda} \frac{\partial}{\partial X_{1}}\right) \bar{E}(X ; \bar{\lambda})=\delta
$$

where

$$
\bar{E}(X ; \bar{\lambda})=\sqrt{1-\mu} E^{*}(x(X) ; \lambda), \quad \Delta=\Delta_{X}, \quad \delta=\delta(X) .
$$

This problem has the well-known fundamental solution

$$
\begin{cases}-\frac{1}{2 \pi} K_{0}\left(\frac{\bar{\lambda} R}{2}\right)^{\bar{\lambda} X_{1} / 2} & \text { if } N=2 \\ -\frac{1}{4 \pi} \frac{e^{-\bar{\lambda} s(X) / 2}}{R} & \text { if } N=3\end{cases}
$$

(see [2])

Remark 2.1. By (2.6),

$$
\bar{E}^{*}(X ; \bar{\lambda}) \sim\left\{\begin{array}{ll}
|\bar{\lambda} X|^{-\frac{1}{2}} \mathrm{e}^{-\bar{\lambda} s(X) / 2} & \text { if } N=2 \\
|X|^{-1} \mathrm{e}^{-\bar{\lambda} s(X) / 2} & \text { if } N=3
\end{array} \quad(\text { as } R=|X| \rightarrow \infty) .\right.
$$

Using the change of variables (2.5) we see that

$$
\begin{aligned}
\sqrt{1-\mu} R & \leq|x| \leq R & & \\
\frac{\sqrt{1-\mu}}{2} s(x) & \leq s(X) \leq 2 s(x) & & \text { if } x_{1} \geq 0 \\
\frac{1}{2} s(x) & \leq s(X) \leq \frac{2}{\sqrt{1-\mu}} s(x) & & \text { if } x_{1} \leq 0
\end{aligned}
$$


with $s(X)=R-X_{1}$. Thus for the study of the asymptotic behaviour, it is not necessary to distinguish between the behaviour in the variables $x$ and $X$.

From (2.7) and Lemma 2.1, setting $r=|x|$, we see:

For $N=2$,

$$
\begin{aligned}
E^{*} \in L^{p}\left(\mathbb{R}^{2}\right) & \text { for } p \in(3, \infty) \\
\partial_{1} E^{*} \in L^{p}\left(\mathbb{R}^{2}\right) & \text { for } p \in(1,2)
\end{aligned}
$$

since

$$
E^{*} \sim\left\{\begin{array}{ll}
r^{-\frac{1}{2}} e^{-s(x)} & (r \rightarrow \infty) \\
\ln r & (r \rightarrow 0)
\end{array}, \quad \partial_{1} E^{*} \sim r^{-\frac{3}{2}} e^{-s(x)}(r \rightarrow \infty), \quad \nabla E^{*} \sim r^{-1}(r \rightarrow 0) .\right.
$$

For $N=3$,

$$
\begin{aligned}
E^{*} \in L^{p}\left(\mathbb{R}^{3}\right) & \text { for } p \in(2,3) \\
\partial_{1} E^{*} \in L^{p}\left(\mathbb{R}^{3}\right) & \text { for } p \in\left(1, \frac{3}{2}\right)
\end{aligned}
$$

since

$$
E^{*} \sim\left\{\begin{array}{ll}
r^{-1} e^{-s} & (r \rightarrow \infty) \\
r^{-1} & (r \rightarrow 0)
\end{array} \quad, \quad \partial_{1} E^{*} \sim r^{-2} e^{-s}(r \rightarrow \infty), \quad \nabla E^{*} \sim r^{-2}(r \rightarrow 0) .\right.
$$

Lemma 2.2. The solution $E^{\mu}=\left(E_{i, j}^{\mu}\right)_{i, j=1}^{N}$ to $(2.2)$ can be expressed in the form

$$
E^{\mu}(x ; \lambda)=\mu \int_{\mathbb{R}^{N}} \frac{\partial}{\partial x_{1}} E^{*}(x-y ; \lambda) \frac{\partial}{\partial y_{1}} \mathcal{O}(y ; \lambda) d y .
$$

This convolution can be understood in the usual notion of $L^{p}$-spaces yielding for every $\lambda>0$

$$
E^{\mu} \in \begin{cases}L^{p}\left(\mathbb{R}^{2}\right) & \text { for } p \in(1, \infty) \\ L^{p}\left(\mathbb{R}^{3}\right) & \text { for } p \in(1,3) .\end{cases}
$$

Furthermore, $E_{j}^{\mu}=\left(E_{i j}^{\mu}\right)_{i=1}^{N}$ satisfies in the sense of Schwartz distributions

$$
\operatorname{div} E_{j}^{\mu}=0 \quad \text { for } j=1, \ldots, N .
$$

Proof. The integrability properties of $\partial_{1} E^{*}$ and $\partial_{1} \mathcal{O}$ and Young's inequality imply that $\partial_{1} E^{*} * \partial_{1} \mathcal{O} \in L^{p}\left(\mathbb{R}^{N}\right)$ with $p \in(1, \infty)$ when $N=2$ and $p \in(1,3)$ when $N=3$. In particular, $E^{\mu}$ can be considered as a distribution in the Schwartz' class $\mathcal{S}^{\prime}$.

Let $\left(\varphi_{k}\right) \subset C_{0}^{\infty}\left(\mathbb{R}^{N}\right)$ be defined by $\varphi_{k}(x)=\varphi_{1}\left(\frac{x}{k}\right)$ where $\varphi_{1}=1$ in the ball $B_{1} \subset \mathbb{R}^{N}$ of radius 1 with center 0 and $\varphi_{1}=0$ in $B_{2}^{c}$. Then elementary $L^{q}$-estimates prove that in the sense of $\mathcal{S}^{\prime}$

$$
\partial_{1} E^{*} * \partial_{1}\left(\varphi_{k} \mathcal{O}\right) \rightarrow \partial_{1} E^{*} * \partial_{1} \mathcal{O} \quad \text { as } k \rightarrow \infty .
$$

Furthermore, defining the operator $A=\Delta-\mu \partial_{1}^{2}-\lambda \partial_{1}$,

$$
\begin{aligned}
A\left(\partial_{1} E^{*} * \partial_{1}\left(\varphi_{k} \mathcal{O}\right)\right) & =\partial_{1}\left(A E^{*}\right) * \partial_{1}\left(\varphi_{k} \mathcal{O}\right) \\
& =\left(A E^{*}\right) * \partial_{1}^{2}\left(\varphi_{k} \mathcal{O}\right) \\
& =\delta * \partial_{1}^{2}\left(\varphi_{k} \mathcal{O}\right) \\
& =\partial_{1}^{2}\left(\varphi_{k} \mathcal{O}\right) \\
& \rightarrow \partial_{1}^{2} \mathcal{O}
\end{aligned}
$$


in the sense of $\mathcal{S}^{\prime}$. Consequently, $A\left(\partial_{1} E^{*} * \partial_{1} \mathcal{O}\right)=\partial_{1}^{2} \mathcal{O}$ in $\mathcal{S}^{\prime}$ proving that $E^{\mu}(\cdot ; \lambda)$ given by $(2.8)$ is a solution of $(2.2)$.

To prove $\operatorname{div} E_{j}^{\mu}=0$ we use Fourier transform in $\mathcal{S}^{\prime}$ to conclude from (2.2) that

$$
\left(-|\xi|^{2}+\mu \xi_{1}^{2}-i \lambda \xi_{1}\right) \widehat{E_{k j}^{\mu}}=-\mu \xi_{1}^{2} \widehat{\mathcal{O}_{k j}}
$$

Since $\operatorname{div} \mathcal{O}_{j}=0$ or, equivalently, $\xi \cdot \widehat{\mathcal{O}_{j}}=0$, we get

$$
\left(-|\xi|^{2}+\mu \xi_{1}^{2}-i \lambda \xi_{1}\right) \mathcal{F}\left(\operatorname{div} E_{j}^{\mu}\right)=0
$$

Consequently, $\operatorname{supp} \mathcal{F}\left(\operatorname{div} E_{j}^{\mu}\right)=\{0\}$ implying that $\operatorname{div} E_{j}^{\mu}$ is a polynomial on $\mathbb{R}^{N}$. However, since $E_{k j}^{\mu} \in L^{p}\left(\mathbb{R}^{N}\right)$ for some $p>1$,

$$
\int_{B_{1}(x)}\left(1-|x-y|^{2}\right) \operatorname{div} E_{j}^{\mu}(y) d y=-2 \int_{B_{1}(x)}(x-y) \cdot E_{j}^{\mu}(y) d y \rightarrow 0
$$

as $|x| \rightarrow \infty$ by Lebesgue's theorem on dominated convergence. Thus the polynomial $\operatorname{div} E_{j}^{\mu}$ vanishes identically in $\mathbb{R}^{N}$

\section{Asymptotic properties of the fundamental solution}

We start to study the asymptotic behaviour of $E^{\mu}$ near infinity. For notational convenience, we define

$$
\eta_{b}^{a}(x)=(1+|x|)^{a}(1+s(x))^{b} .
$$

We have (again, as for the classical Oseen problem, we put $\lambda=1$ )

Lemma 3.1. Let $N=3$. Then, for $k \geq 0, \varepsilon>0$ arbitrarily small and $|x| \geq R \gg 1$,

$$
\left|\nabla^{k} E_{i j}^{\mu}(x ; 1)\right| \leq C|x|^{-\frac{4+k-\varepsilon}{2}}(1+s(x))^{-\frac{2+k}{2}} .
$$

Proof. We divide the convolution integral (2.8) into three parts:

$$
\begin{aligned}
E_{i j}^{\mu}(x ; 1) & =\mu\left(\int_{B_{1}(0)}+\int_{B_{1}(x)}+\int_{\mathbb{R}^{3} \backslash B_{1}(0) \backslash B_{1}(x)}\right) \frac{\partial E^{*}(x-y ; 1)}{\partial x_{1}} \frac{\partial O_{i j}(y ; 1)}{\partial y_{1}} d y \\
& \equiv I_{1}(x)+I_{2}(x)+I_{3}(x) .
\end{aligned}
$$

Obviously,

$$
\left|I_{1}(x)\right|+\left|I_{2}(x)\right| \leq C \eta_{-1}^{-2}(x)
$$

for $|x|$ sufficiently large. For $I_{3}$ it is enough to estimate the convolution

$$
\left|I_{3}(x)\right| \leq C \int_{\mathbb{R}^{3}}(1+|x-y|)^{-2} e^{-s(x-y)} \eta_{-1}^{-2}(y) d y
$$


which can be assumed as $\left(\eta_{-m}^{-2} * \eta_{-1}^{-2}\right)(x)$ for $m$ arbitrarily large. We shortly sketch how to estimate such convolutions (for more details see, e.g., [1,3] or [6]). Let $x^{\prime}=\left(x_{2}, \ldots, x_{N}\right)$. We distinguish the following four situations:
a) $|x| \leq R_{0}$
b) $x_{1}>0,\left|x^{\prime}\right|<\sqrt{x_{1}},|x|>R_{0}$
c) $x_{1}>0,\left|x^{\prime}\right|=\frac{1}{2}|x|^{\frac{1}{2}+\sigma} \quad\left(\sigma \in\left[0, \frac{1}{2}\right],|x|>R_{0}\right)$
d) $x_{1}>0,\left|x^{\prime}\right|>\frac{|x|}{2},|x|>R_{0}$ or $x_{1}<0,|x|>R_{0}$.

The most restrictive case is c). Here we divide $\mathbb{R}^{N}$ into 16 subdomains, as shown in Figure 1 below. Here $R=|x|$ and $\nu=\sigma+\frac{1}{2}$.

Figure 1

Now we calculate the convolutions over each subdomain $\Omega_{i}(i=0,1, \ldots, 15)$. After some lengthy computations we get exponents $e_{i}, f_{i} \in \mathbb{R}$ such that

$$
\int_{\Omega_{i}} \eta_{-d}^{-c}(x-y) \eta_{-b}^{-a}(y) d y \leq C \eta_{-f_{i}}^{-e_{i}}(x) .
$$

Now, taking $e \leq \min \left\{e_{i}\right\}_{i=0}^{15}$ and $f \leq \min \left\{f_{i}\right\}_{i=0}^{15}$, we obtain the desired inequality $\left(\eta_{-d}^{-c} * \eta_{-b}^{-a}\right)(x) \leq C \eta_{-f}^{-e}(x)$. The situation is slightly more complicated by the presence of some logarithmic terms which can be absorbed by taking $e<\min \left\{e_{i}\right\}_{i=0}^{15}$ and/or $f<\min \left\{f_{i}\right\}_{i=0}^{15}$. The calculations are quite technical and the results are summarized in [1] when $N=3$ and in Tables $1-4$ in [3] or [6] for $N=2,3$; the results for $N>3$ can be read from the calculations performed in the above references. Thus we get

$$
\left|I_{3}(x)\right| \leq C \eta_{-1}^{-2+\varepsilon}(x)
$$


where the $\varepsilon$-loss is caused by logarithmic factors. Continuing with the first derivatives we easily observe that

$$
\left|\frac{\partial}{\partial x_{i}} I_{1}(x)\right|+\left|\frac{\partial}{\partial x_{i}} I_{2}(x)\right| \leq C \eta_{-\frac{3}{2}}^{-\frac{5}{2}}(x) .
$$

The term $\frac{\partial}{\partial x_{i}} I_{3}(x)$ needs more care. In subdomains $\Omega_{0}, \Omega_{2}, \Omega_{6}, \Omega_{8}$ and $\Omega_{11}$ (and in the corresponding situations in $\Omega_{4}, \Omega_{9}, \Omega_{10}, \Omega_{14}$ and $\left.\Omega_{15}\right)$ we simply take the derivative of $I_{3}$, otherwise we first use the change of variables $\mathbf{z}=x-y$ and then calculate the derivative. Using Tables 1 - 2 from [3] or [6] we again obtain

$$
\left|\frac{\partial}{\partial x_{i}} I_{3}(x)\right| \leq C \eta_{-\frac{3}{2}}^{-\frac{5}{2}+\varepsilon}(x)
$$

Analogously we proceed for higher derivatives

Similarly, in two space dimensions:

Lemma 3.2. Let $N=2$. Then, for $k \geq 0, \varepsilon>0$ arbitrarily small and $|x| \geq R \gg 1$,

$$
\left|\nabla^{k} E_{i j}^{\mu}(x ; 1)\right| \leq C|x|^{-\frac{3+k-\varepsilon}{2}}(1+s(x))^{-\frac{1+k}{2}} .
$$

Proof. It is analogous to the three-dimensional case

Remark 3.1. Obviously, $E^{\mu}$ and $\nabla E^{\mu}$ decay faster than $\mathcal{O}$ or $\nabla \mathcal{O}$, respectively. Only for $\nabla^{2} E^{\mu}$, due to a certain $\varepsilon$-loss, $\frac{\partial^{2} \mathcal{O}}{\partial x_{1}^{2}}$ decays faster near the axis $x_{1}$. Nevertheless, it can be shown that the logarithmic factor does not appear and that $\frac{\partial^{2} E^{\mu}}{\partial x_{1}^{2}}$ decays as fast as $\frac{\partial^{2} \mathcal{O}}{\partial x_{1}^{2}}$. Note also that in applications we usually do not distinguish the asymptotic behaviour of particular components of $\nabla^{2} \mathcal{O}$.

The investigation of $E^{\mu}$ near zero is more delicate. We wish to verify not only that $\nabla^{2} E^{\mu} \sim|x|^{-N}$ for $|x| \rightarrow 0$, but also that the singular part of $\nabla^{2} E^{\mu}$ represents, similarly as $\nabla^{2} \mathcal{S}$, a kernel of a bounded operator from $L^{p}\left(\mathbb{R}^{N}\right)$ into $L^{p}\left(\mathbb{R}^{N}\right)$.

For simplicity we omit in the following the parameter $\lambda=1$ in (2.8). Introduce two cut-off functions $\varphi, \psi \in C_{0}^{\infty}\left(\mathbb{R}^{N}\right)$ satisfying

$$
\varphi=\left\{\begin{array}{ll}
1 & \text { on } B_{2} \\
0 & \text { on } B_{3}^{c}
\end{array} \quad \text { and } \quad \psi= \begin{cases}1 & \text { on } B_{4} \\
0 & \text { on } B_{5}^{c} .\end{cases}\right.
$$

Then

$$
E^{\mu}=\mu \partial_{1} E^{*} * \partial_{1} \mathcal{O}=I_{1}+I_{2}+I_{3}
$$

where

$$
I_{1}(x)=\mu \int \partial_{1}\left(\psi E^{*}\right)(x-y) \partial_{1}(\varphi \mathcal{O})(y) d y
$$

is the most crucial term,

$$
I_{2}=\mu \partial_{1}\left((1-\psi) E^{*}\right) * \partial_{1}(\varphi \mathcal{O})
$$


vanishes in $B_{1}$, and

$$
I_{3}=\mu \partial_{1} E^{*} * \partial_{1}((1-\varphi) \mathcal{O})
$$

is easily seen to be bounded together with all its derivatives in $B_{1}$. Obviously,

$$
J=I_{1}+I_{2}=\mu \partial_{1} E^{*} * \partial_{1}(\varphi \mathcal{O})
$$

satisfies

$$
\left(\Delta-\mu \partial_{1}^{2}-\partial_{1}\right) J=\mu \partial_{1}^{2}(\varphi \mathcal{O}) \quad \text { in } \mathcal{S}^{\prime}
$$

(cf. Lemma 2.2). Since $I_{2}=0$ in $B_{1}$, we conclude that $I_{1} \in L^{p}\left(B_{1}\right) \cap C^{\infty}\left(B_{1} \backslash\{0\}\right)$ where $p \in(1, \infty)$ for $N=2$ and $p \in(1,3)$ for $N=3$.

To establish the singular behaviour of $I_{1}$ near 0 we estimate its Fourier transform

$$
\hat{I}_{1}=(2 \pi)^{\frac{N}{2}} \mu \mathcal{F}\left(\partial_{1}\left(\psi E^{*}\right)\right) \cdot \mathcal{F}\left(\partial_{1}(\psi \mathcal{O})\right)=-(2 \pi)^{\frac{N}{2}} \mu \xi_{1}^{2} \widehat{\psi E^{*}} \cdot \widehat{\varphi \mathcal{O}}
$$

and the $\xi$-derivative $D_{\xi}^{\alpha} \hat{I}_{1}$. Note that this formula for $\hat{I}_{1}$ is justified by [7: Theorem 30.4]. Since $x^{\alpha} \partial_{1}\left(\psi E^{*}\right), x^{\alpha} \partial_{1}(\varphi \mathcal{O}) \in L^{1}\left(\mathbb{R}^{N}\right)$ for all $\alpha \in \mathbb{N}_{0}^{N}$, the functions $D_{\xi}^{\alpha} \mathcal{F}\left(\partial_{1}\left(\psi E^{*}\right)\right)$ and $D_{\xi}^{\alpha} \mathcal{F}\left(\partial_{1}(\varphi \mathcal{O})\right)$ are continuous on $\mathbb{R}^{N}$ vanishing for $|\xi| \rightarrow \infty$, the same result being true when the partial derivative $\partial_{1}$ is omitted. Thus for all $\alpha \in \mathbb{N}_{0}^{N}$

$$
D_{\xi}^{\alpha} \hat{I}_{1}(\xi)=O\left(|\xi|^{2-|\alpha|}\right) \quad \text { for }|\xi| \rightarrow 0 .
$$

For large $|\xi|$ we use the representation (see [7: Theorem 30.4])

$$
\widehat{\psi E^{*}}(\xi)=(2 \pi)^{-\frac{N}{2}} \int_{R^{N}} \hat{\psi}(\xi-\lambda) \widehat{E^{*}}(\lambda) d \lambda
$$

where $\hat{\psi} \in \mathcal{S}$ and

$$
\left|\widehat{E^{*}}(\lambda)\right| \leq \frac{c}{|\lambda|^{2}+\left|\lambda_{1}\right|} \leq \frac{c}{\left|\lambda_{1}\right|^{\frac{2}{3}}\left|\lambda^{\prime}\right|^{\frac{2}{3}}} \quad\left(\lambda=\left(\lambda_{1}, \lambda^{\prime}\right)\right) .
$$

Consequently,

$$
\begin{aligned}
\left|\widehat{\psi E^{*}}(\xi)\right| & \leq \frac{c_{M}}{|\xi|^{M}} \int_{|\lambda| \leq \frac{|\xi|}{2}} \frac{1}{\left|\lambda_{1}\right|^{\frac{2}{3}}\left|\lambda^{\prime}\right|^{\frac{2}{3}}} d \lambda+\frac{c_{M}}{|\xi|^{2}} \int_{|\lambda|>\frac{|\xi|}{2}} \frac{1}{(1+|\xi-\lambda|)^{M}} d \lambda \\
& \leq c\left(|\xi|^{-M+N+-\frac{4}{3}}+|\xi|^{-2}\right)
\end{aligned}
$$

for $M \in \mathbb{N}$ arbitrarily large. Hence, dealing analogously with $\widehat{\varphi \mathcal{O}}$,

$$
\left|\widehat{\psi E^{*}}(\xi)\right|+\widehat{\varphi \mathcal{O}}(\xi) \mid=O\left(|\xi|^{-2}\right) \quad \text { as }|\xi| \rightarrow \infty .
$$

For the partial derivative

$$
\begin{aligned}
D_{\xi}^{\alpha} \widehat{\psi E^{*}}(\xi) & =(2 \pi)^{-\frac{N}{2}} \int_{\mathbb{R}^{N}}(-1)^{|\alpha|} D_{\lambda}^{\alpha} \hat{\psi}(\xi-\lambda) \widehat{E^{*}}(\lambda) d \lambda \\
& =\int_{|\lambda| \leq \frac{|\xi|}{2}} \cdots+\int_{|\lambda| \geq \frac{|\xi|}{2}} \cdots
\end{aligned}
$$


we use integration by parts $|\alpha|$-times in the second integral. Here the first integral on $|\lambda| \leq \frac{|\xi|}{2}$ and $|\alpha|$-many boundary integrals on $|\lambda|=\frac{|\xi|}{2}$ can be estimated by $c_{M}|\xi|^{-M}$ for $M$ arbitrarily large. Finally, for the remaining integral,

$$
\begin{aligned}
\left|\int_{|\lambda| \geq \frac{|\xi|}{2}} \hat{\psi}(\xi-\lambda) D_{\lambda}^{\alpha} \widehat{E}^{*}(\lambda) d \lambda\right| & \leq c_{M} \int_{|\lambda| \geq \frac{|\xi|}{2}} \frac{1}{(1+|\xi-\lambda|)^{M}}|\xi|^{-2-|\alpha|} d \lambda \\
& \leq c|\xi|^{-2-|\alpha|} .
\end{aligned}
$$

Thus we proved that for all $\alpha \in \mathbb{N}_{0}^{n}$

$$
\left|D_{\xi}^{\alpha} \widehat{\psi E^{*}}(\xi)\right|+\left|D_{\xi}^{\alpha} \widehat{\varphi \mathcal{O}}(\xi)\right|=O\left(|\xi|^{-2-|\alpha|}\right) \quad \text { as }|\xi| \rightarrow \infty .
$$

Summarizing (3.3) and (3.4) we obtain

$$
\left|D_{\xi}^{\alpha} \hat{I}_{1}(\xi)\right| \leq c_{\alpha} \frac{|\xi|^{2-|\alpha|}}{(1+|\xi|)^{4}} \quad \text { for } \xi \neq 0, \alpha \in \mathbb{N}_{0}^{N} .
$$

We shall now reconstruct the asymptotic properties of $I_{1}(x)$ using the following lemma.

Lemma 3.3. Let $\mathcal{F}(G) \in C^{m+N-1}\left(\mathbb{R}^{N} \backslash\{0\}\right) \quad(N \geq 2)$ be such that

$$
A:=\sup _{\substack{|\alpha| \leq m+N-1 \\ \xi \in \mathbb{R}^{N} \backslash\{\mathbf{0}\}}}(1+|\xi|)^{4}|\xi|^{|\alpha|-2}\left|D^{\alpha} \mathcal{F}(G)(\xi)\right|<\infty .
$$

Then $G \in C^{m}\left(\mathbb{R}^{N} \backslash\{0\}\right)$ and there exists $c_{m, N}>0$ such that for all $0 \neq x \in B_{1}$

$$
\begin{aligned}
& |G(x)| \leq \begin{cases}c_{m, N} A|\ln | x|| & \text { if } N=2 \\
c_{m, N} A|x|^{2-N} & \text { if } N \geq 3\end{cases} \\
& \left|D^{\beta} G(x)\right| \leq c_{m, N} A|x|^{2-N-|\beta|} \quad \text { if } N \geq 2,0<|\beta| \leq m \text {. }
\end{aligned}
$$

Furthermore, for $x \in B_{1}^{c}, N \geq 2$ and $0 \leq|\beta| \leq m$,

$$
\left|D^{\beta} G(x)\right| \leq c_{m, N} A|x|^{1-N-|\beta|} .
$$

Proof. Fix $\Phi \in C_{0}^{\infty}\left(\mathbb{R}^{N}\right)$ such that $\Phi=1$ in $B_{\frac{1}{2}}$ and $\Phi=0$ in $B_{1}^{c}$. For $\beta \in \mathbb{N}_{0}^{N}$ and $\lambda>0$ we define $v_{\lambda, \beta}$ and $w_{\lambda, \beta}$ by

$$
\begin{aligned}
\mathcal{F}\left(v_{\lambda, \beta}\right)(\xi) & =(i \xi)^{\beta} \widehat{G}(\xi) \Phi\left(\frac{\xi}{\lambda}\right) \\
\mathcal{F}\left(w_{\lambda, \beta}\right)(\xi) & =(i \xi)^{\beta} \widehat{G}(\xi)\left(1-\Phi\left(\frac{\xi}{\lambda}\right)\right)
\end{aligned}
$$

yielding

$$
\mathcal{F}\left(v_{\lambda, \beta}\right)+\mathcal{F}\left(w_{\lambda, \beta}\right)=(i \xi)^{\beta} \widehat{G}(\xi)=\mathcal{F}\left(D^{\beta} G\right)(\xi) .
$$


Moreover, $\mathcal{F}\left(v_{\lambda, \beta}\right) \in L^{1}\left(\mathbb{R}^{N}\right)$ and therefore

$$
\left|v_{\lambda, \beta}(x)\right| \leq c A \int_{|\xi| \leq \lambda}|\xi|^{|\beta|+2}(1+|\xi|)^{-4} d \xi \leq c A \int_{0}^{\lambda} \frac{r^{|\beta|+N+1}}{(1+r)^{4}} d r
$$

For $\lambda<1$ we get $\left|v_{\lambda, \beta}(x)\right| \leq c A \lambda^{|\beta|+N+2}$, while for $\lambda>1$ we distinguish between the cases $\beta=0, N=2$ and $|\beta|+N>2$. Obviously,

$$
\begin{array}{ll}
\left|v_{\lambda, 0}(x)\right| \leq c A(1+\ln \lambda) & \text { if } N=2 \\
\left|v_{\lambda, \beta}(x)\right| \leq c A \lambda^{|\beta|+N-2} & \text { if }|\beta|+N>2 .
\end{array}
$$

Now choose $p \in \mathbb{N}_{0}^{N}$ such that $|p|=|\beta|+N-1$. We will show that $x^{p} w_{\lambda, \beta}(x)$ is a continuous function tending to 0 as $|x| \rightarrow \infty$, satisfying

$$
\left|x^{p} w_{\lambda, \beta}(x)\right| \leq c A .
$$

The function $\mathcal{F}\left(w_{\lambda, \beta}\right)$ has its support in $B_{\lambda / 2}^{c}$. Evidently, $D^{p} \mathcal{F}\left(w_{\lambda, \beta}\right) \in L^{1}\left(\mathbb{R}^{N}\right)$ since it is a linear combination of

$$
\xi^{\beta-q} D^{\alpha} \widehat{G}(\xi) D^{r}\left(1-\Phi\left(\frac{\xi}{\lambda}\right)\right)
$$

where $\alpha+q+r=p$ and $q \leq \beta$. If $r \neq 0$, its support lies between two spheres with diameters $\frac{\lambda}{2}$ and $\lambda$. Therefore

$$
\begin{aligned}
c \lambda^{-|r|} & \int_{\frac{\lambda}{2}<|\xi|<\lambda}|\xi|^{|\beta|-|q|}\left|D^{\alpha} \widehat{G}(\xi)\right| d \xi \\
\leq & c A \lambda^{-|r|} \int_{\frac{\lambda}{2}<|\xi|<\lambda}|\xi|^{|\beta|-|q|+2-|\alpha|}(1+|\xi|)^{-4} d \xi \\
& \leq c A \lambda^{-|p|+|\beta|+N+2}(1+\lambda)^{-4} .
\end{aligned}
$$

If $r=0$, then the support of $\xi^{\beta-q} D^{\alpha} \widehat{G}(\xi)\left(1-\Phi\left(\frac{\xi}{\lambda}\right)\right)$ lies in $B_{\lambda / 2}^{c}$ and

$$
\begin{aligned}
c A \int_{|\xi| \geq \frac{\lambda}{2}}|\xi|^{|\beta|-|q|+2-|\alpha|}(1+|\xi|)^{-4} d \xi & \leq c A \int_{\frac{\lambda}{2}}^{\infty} r^{|\beta|-|p|+N+1}(1+r)^{-4} d r \\
& \leq c A(1+\lambda)^{-1} .
\end{aligned}
$$

Summarizing the different estimates of $x^{p} w_{\lambda, \beta}(x)$ we obtain

$$
|x|^{|p|}\left|w_{\lambda, \beta}(x)\right| \leq c A \lambda^{-|p|+|\beta|+N-1}(1+\lambda)^{-1} .
$$

Setting $\lambda=\frac{1}{|x|}$ we get the result 
In particular, we have reproved that

$$
I_{1}(x) \sim\left\{\begin{array}{ll}
\ln |x| & \text { if } N=2 \\
\frac{1}{|x|} & \text { if } N=3
\end{array} \quad(|x| \rightarrow 0) .\right.
$$

Moreover, we have obtained that for $0<|\beta| \leq 2$

$$
D^{\beta} I_{1}(x) \sim \frac{1}{|x|^{|\beta|+N-2}} \quad \text { in } B_{1} .
$$

Therefore the integral operator

$$
\int_{\mathbb{R}^{N}} \frac{\partial I_{1}}{\partial x_{i}}(x-y) f(y) d y
$$

is well defined on $C_{0}^{\infty}\left(\mathbb{R}^{N}\right)$ and can be (eventually) extended due to a density argument onto some $L^{q}\left(\mathbb{R}^{N}\right)$. Let us consider the integral operator

$$
T f(x)=\int_{\mathbb{R}^{N}} I_{1}(x-y) \frac{\partial^{2} f(y)}{\partial y_{i} \partial y_{j}} d y
$$

for $f \in C_{0}^{\infty}\left(\mathbb{R}^{N}\right)$. Evidently,

$$
\mathcal{F}(T f)(\xi)=(2 \pi)^{\frac{N}{2}} \hat{I}_{1}(\xi)\left(-\xi_{i} \xi_{j}\right) \hat{f}(\xi)
$$

where the multiplication is to be understood in the sense of $\mathcal{S}^{\prime}$. Nevertheless, thanks to the properties of $\hat{I}_{1}(\xi)$ we easily see that

$$
\mathcal{F}(T f)(\xi)=-(2 \pi)^{\frac{N}{2}} \xi_{i} \xi_{j} \hat{I}_{1}(\xi) \hat{f}(\xi)=m(\xi) \hat{f}(\xi)
$$

where $\left.m(\xi) \in L^{\infty}\left(\mathbb{R}^{N}\right) \cap C^{\infty}\left(\mathbb{R}^{N}\right) \backslash\{0\}\right)$ and

$$
\left|D^{\alpha} m(\xi)\right| \leq \frac{C}{|\xi|^{|\alpha|}} \quad \text { for all } \xi \neq 0, \alpha \in \mathbb{N}_{0}^{n}
$$

Therefore, $m(\xi)$ is an $L^{p}$-multiplier, $1<p<\infty$. Thus we get the following lemma.

Lemma 3.4. Let $0<|\alpha| \leq 2$. Then we have for $|x| \leq 1$

$$
\left|E_{i j}^{\mu}(x ; 1)\right| \leq\left\{\begin{array}{ll}
C \ln |x| & \text { if } N=2 \\
C|x|^{-1} & \text { if } N=3
\end{array} \quad \text { and } \quad\left|D^{\alpha} E_{i j}^{\mu}(x ; 1)\right| \leq C|x|^{-|\alpha|-N+2} .\right.
$$

Moreover,

$$
E_{i j}^{\mu}(x ; 1)=I_{1}(x)+I_{2}(x)
$$

where $\left|D^{\alpha} I_{2}(x)\right| \leq C$ for $|x| \leq 1,|\alpha|=2$, and $I_{1}(x)$, representing the singular part of the second gradient of $E_{i j}^{\mu}(x ; 1)$, has the property

$$
\mathcal{F}\left(\int_{\mathbb{R}^{N}} I_{1}(\cdot-y) D^{\alpha} f(y) d y\right)(\xi)=m(\xi) \hat{f}(\xi) \quad(|\alpha|=2)
$$


where $m(\xi)$ represents an $L^{p}$-Fourier multiplier, $1<p<\infty$. Therefore, if $|\alpha|=2$, the integral operator $T$,

$$
T f(x)=\int_{\mathbb{R}^{N}} I_{1}(x-y) D^{\alpha} f(y) d y,
$$

maps $C_{0}^{\infty}\left(\mathbb{R}^{N}\right)$ into $L^{p}\left(\mathbb{R}^{N}\right) \quad(1<p<\infty)$ and

$$
\begin{aligned}
\|T f\|_{p, \mathbb{R}^{N}} & \leq C\|f\|_{p, \mathbb{R}^{N}} \\
\|T f\|_{p,(g), \mathbb{R}^{N}} & \leq C\|f\|_{p,(g), \mathbb{R}^{N}}
\end{aligned}
$$

for all weights $g$ from the Muckenhoupt class $A_{p}$.

Remark 3.2. The non-negative weight $g$ belongs to the Muckenhoupt class $A_{r}(1 \leq$ $r<+\infty)$ if there is a constant $C$ such that

$$
\sup _{Q}\left[\left(\frac{1}{|Q|} \int_{Q} g(x) d x\right)\left(\frac{1}{|Q|} \int_{Q} g(x)^{-\frac{1}{r-1}} d x\right)^{r-1}\right] \leq C<\infty
$$

if $r \in(1, \infty)$ and

$$
\sup _{Q} \frac{1}{|Q|} \int_{Q} g(x) d x \leq C g\left(x_{0}\right) \quad \forall x_{0} \in \mathbb{R}^{N}
$$

if $r=1$. In the first case, the supremum is taken over all cubes $Q$ in $\mathbb{R}^{N}$, in the second case only over those cubes which contain $x_{0} ;|Q|$ denotes the Lebesgue measure of $Q$. The constant does not depend on $x_{0}$.

Finally, we study how

$$
E_{i j}^{\mu}(x ; \lambda)=\mu \int_{\mathbb{R}^{N}} \frac{\partial E^{*}(x-y ; \lambda)}{\partial x_{1}} \frac{\partial \mathcal{O}_{i j}(y ; \lambda)}{\partial y_{1}} d y
$$

behaves for $\lambda \neq 1$. Recall that we usually assume $\lambda \ll 1$. Since

$$
\begin{aligned}
\mathcal{O}_{i j}(x ; \lambda) & =\lambda^{N-2} \mathcal{O}_{i j}(\lambda x ; 1) \\
E^{*}(x ; \lambda) & =\lambda^{N-2} E^{*}(\lambda x ; 1)
\end{aligned}
$$

we get

$$
E_{i j}^{\mu}(x ; \lambda)=\lambda^{N-2} E_{i j}^{\mu}(\lambda x ; 1)
$$

We can now summarize the results.

Theorem 3.1. Let $N=2,3$ and $0 \leq \mu<1$. Then the solution to (0.6) can be written in the form

$$
\begin{aligned}
\mathcal{O}^{\mu}(x ; \lambda) & =\mathcal{O}(x ; \lambda)+E^{\mu}(x ; \lambda) \\
e(x) & =\nabla \mathcal{E}(x)
\end{aligned}
$$

here $\mathcal{E}(x)$ denotes the fundamental solution to the Laplace equation, $\mathcal{O}(x ; \lambda)$ is the fundamental Oseen tensor and $E^{\mu}(x ; \lambda)$ has the following properties: 
a) For $|x| \geq R \gg 1,|\alpha| \geq 0$ and $\varepsilon>0$ arbitrarily small

$$
\left|D^{\alpha} E_{i j}^{\mu}(x ; 1)\right| \leq C|x|^{-\frac{N+1+|\alpha|-\varepsilon}{2}}(1+s(x))^{-\frac{N-1+|\alpha|}{2}} .
$$

b) For $|x| \leq 1$ and $|\alpha|=1,2$

$$
\begin{aligned}
& \left|E_{i j}^{\mu}(x ; 1)\right| \leq \begin{cases}C \ln |x| & \text { if } N=2 \\
C|x|^{-1} & \text { if } N=3\end{cases} \\
& \left|D^{\alpha} E_{i j}^{\mu}(x ; 1)\right| \leq C|x|^{2-N-|\alpha|} \text { if } N=2,3 \text {. }
\end{aligned}
$$

Moreover, $E_{i j}^{\mu}(x ; 1)=I_{1}(x)+I_{2}(x)$, where $\left|D^{\alpha} I_{2}(x)\right| \leq C$ for all $|\alpha| \leq 2$ and $|x| \leq 1$, and $D^{\alpha} I_{1}(x),|\alpha|=2$, representing the singular part of the second gradient of $E^{\mu}(x ; 1)$, defines an integral operator

$$
T f(x)=\int_{\mathbb{R}^{N}} I_{1}(x-y) D^{\alpha} f(y) d y \quad(|\alpha|=2)
$$

which maps $L^{p}\left(\mathbb{R}^{N}\right)$ into $L^{p}\left(\mathbb{R}^{N}\right)$ and $\overline{C_{0}^{\infty}\left(\mathbb{R}^{N}\right)}\|\cdot\|_{p,(g)}$ into $L^{p}\left(\mathbb{R}^{N} ; g\right)$ for $1<p<\infty$, $g \in A_{p}$ (Muckenhoupt class). Moreover,

$$
E^{\mu}(x ; \lambda)=\lambda^{N-2} E^{\mu}(\lambda x ; 1) .
$$

\section{References}

[1] Farwig, R.: The stationary exterior 3D-problem of Oseen and Navier-Stokes equations in anisotropically Sobolev spaces. Math. Z. 211 (1992), 409 - 447.

[2] Galdi, G. P.: An Introduction to the Mathematical Theory of the Navier-Stokes Equations I (Springer Tracts in Natural Philosophy: Vol. 38). New York: Springer 1994.

[3] Kračmar, S., Novotný, M. and M. Pokorný: Estimates of Oseen kernels in weighted $L^{p}$ spaces. J. Math. Soc. Japan (submitted).

[4] Novotný, M. and M. Pokorný: Three-dimensional steady flow of viscoelastic fluid past an obstacle. J. Math. Fluid Dynamics (submitted).

[5] Novotný, M. and M. Pokorný: Steady plane flow of viscoelastic fluid past an obstacle. Appl. Math. (submitted).

[6] Pokorný, M.: Asymptotic behaviour of solutions to certain PDE's describing the flow of fluids in unbounded domains. Ph.D. thesis. Prague: Charles University \& Toulon - La Garde: University of Toulon and Var 1999.

[7] Treves, F.: Topological Vector Spaces, Distributions and Kernels. New York: Acad. Press 1967.

Received 31.08.1999; in revised form 07.02.00 